\title{
Inverse Interval Field Quantification via Digital Image Correlation
}

\author{
Matthias Faes ${ }^{1, \mathrm{a}}$, and David Moens ${ }^{1, \mathrm{~b}}$ \\ KU Leuven - Department of Mechanical Engineering, Jan De Nayerlaan 5, 2860 \\ St.-Katelijne-Waver, Belgium \\ aamatthias.faes@kuleuven.be, bdavid.moens@kuleuven.be
}

Keywords: Interval field analysis, inverse uncertainty quantification, multivariate uncertainty

\begin{abstract}
This paper presents the application of a new method for the identification and quantification of interval valued spatial uncertainty under scarce data. Specifically, full-field strain measurements, obtained via Digital Image Correlation, are applied in conjunction with a quasi-static finite element model. To apply these high-dimensional but scarce data, extensions to the novel method are introduced. A case study, investigating spatial uncertainty in Young's modulus of PA-12 parts, produced via Laser Sintering, shows that an accurate quantification of the constituting uncertainty is possible, albeit being somewhat conservative with respect to deterministic values reported in literature.
\end{abstract}

\section{Introduction}

They key objective of a modern design engineer is to develop a deterministic structure that is reliable in a non-deterministic environment. For example, when designing an aircraft wing, a large amount of uncertainty is typically present on the crisp parameters of the constitutive models of the composite materials, but also concerning the exact loading that this wing has to withstand during its entire service life time. Classically, a semi-heuristic approach is applied, where a deterministic scaling of the design is performed using safety factors to account implicitly for the non-deterministic design quantities. In the context of high-performance structures, this method is questionable since it does not account for the non-determinism explicitly or in a mathematically rigorous way, leading to a highly subjective design and a possible severe misjudgement of reality. To account for this, these safety factors are typically chosen rather large, yielding a design that is very over-conservative, which is impermissible in many applications where the total mass of the structure is critical.

As an alternative approach, advanced techniques for non-deterministic numerical analysis have been introduced in the last few decades. Available tools include methods from the highly matured probabilistic framework (e.g., advanced Monte Carlo methods) [1], possibilistic techniques such as interval, fuzzy or convex arithmetical approaches [2] or techniques based on the concepts of imprecise probability [3]. As concerns intra-domain non-determinism in a probabilistic context, the propagation of the constituting random fields (RF) following a Karhunen-Loève (KL) approach (i.e., based on the spectral decomposition of the covariance kernel), is considered to be highly matured [4]. However, the definition of the covariance kernel at the core of the KL-scheme is often highly non-trivial, whereas it marks a large influence on both the spatial nature and the numerical approximation of the RF. Interval fields (IF), which represent the spatial ND as a finite series expansion about a set of deterministic base functions, weighted with independent interval scalars, were only established very recently as an interval counterpart to RF by the authors [5], and by other research groups [16, 17].

All these highly advanced techniques however require an accurate and realistic representation of the constituting uncertainty to enable their application in realistic design computations. Hence, the non-determinism in the relevant model quantities needs to be quantified, which is often not trivial due to cost-, time- or practical constraints. Such quantification is especially challenging when the uncertain model quantities are not directly measurable, or when their measurement is too expensive to be conducted on a large scale. In this context, inverse uncertainty quantification techniques need to be applied, where the uncertain model parameters are quantified based on a set of measured structural responses and a model that predicts these responses based on the uncertain model parameters. In 
a probabilistic context, such inverse quantification is usually performed in a Bayesian context [9, 10]. However, these techniques typically require an elaborate data set to obtain an objective posterior distribution of the non-deterministic parameters. The quantification of scalar interval uncertainty is usually obtained by minimizing a squared $L_{2}$ norm over the difference between the interval bounds of the measurement data and interval finite element model [6, 7] or other based on Kriging surfaces [8]. However, as this approach neglects dependence between individual model responses, it is not possible to quantify spatial interval uncertainty. Therefore, techniques for the inverse quantification of interval fields on the other hand were only introduced very recently by the authors $[11,12,13]$, and require very few data to obtain an objective quantification of the constituting uncertainty.

This paper applies these new inverse methods for interval field quantification to the specific case of quantifying spatial uncertainty in a quasi-static testing environment using Digital Image Correlation. To apply this contactless strain measurement technique in the novel interval field quantification procedure, some additional steps in the data preprocessing are needed, which are also presented. Specifically, a case study concerning spatial uncertainty in Laser Sintered components is considered.

\section{Inverse Interval Field Finite Element Analysis.}

Let $\mathcal{M}$ be a deterministic Finite Element model that is used to solve a (set of) differential equations for some model responses $\boldsymbol{y}^{m} \in \mathbb{R}^{d}$ (e.g., modal responses or strains) through the vector valued function operator $m$ :

$$
\mathcal{M}(\boldsymbol{x}): y_{i}^{m}=m_{i}(\boldsymbol{x}), \quad m_{i}: \mathbb{R}^{k} \mapsto \mathbb{R}, \quad i=1, \ldots, d
$$

with $\boldsymbol{x} \in \mathcal{F} \subset \mathbb{R}^{k}$ the vector of model parameters and $\mathcal{F}$ the sub-domain of feasible parameters (e.g., non-negative contact stiffness). The uncertainty that is attributed to $\boldsymbol{x}$ is modelled as an interval vector $\boldsymbol{x}^{I} \in \mathcal{F}^{I} \subset \mathbb{R}^{k}$, with $\mathbb{I}^{k}$ the space of $k$-dimensional interval vectors. Note that due the orthogonality of all $x_{i}^{I}, i=1, \ldots, k$, all model parameters are considered independent by definition. Therefore, $\boldsymbol{x}^{I}$ can also be represented as a $k$-dimensional hypercube.

In case $\boldsymbol{x}(\boldsymbol{r})$, with $\boldsymbol{r} \in \Omega \subset \mathbb{R}^{\omega}$ and $\omega \leq 4$, is a function over the model domain $\Omega$, a pure hyper-cubic representation of the uncertain parameter (e.g., by assigning an interval to each element in the discretised $\Omega$ ) yields an intractable computational cost, as well as non-physical realisations. The former is a direct result of the so-called "curse of dimensionality", whereas the latter stems from the orthogonality by construction between the locally defined intervals [12, 13]. As a remediation, the interval field concept was recently introduced by the authors [5]. An interval field $\boldsymbol{x}^{I}(\boldsymbol{r})$ models the spatial uncertain parameter as a superposition of orthonormal base functions $\boldsymbol{\psi}_{i}(\boldsymbol{r}): \Omega \mapsto(0,1]$, scaled by independent interval scalars $\boldsymbol{\alpha}^{\boldsymbol{I}}=\left[\alpha_{1}^{I}, \alpha_{2}^{I}, \ldots, \alpha_{n_{b}}^{I}\right]$ :

$$
\boldsymbol{x}^{I}(\boldsymbol{r})=\sum_{i=1}^{n_{b}} \boldsymbol{\psi}_{i}(\boldsymbol{r}) \cdot \alpha_{i}^{I}
$$

These base functions can e.g., be constructed using inverse distance weighting interpolation [13]. As such, the uncertainty is captured by the interval scalars $\boldsymbol{\alpha}^{I}$, whereas the spatial nature of the uncertainty is modelled by the basis functions. Hence, the uncertainty remains captured inside a hyper-cubic set, which facilitates the uncertainty propagation, whereas at the element level, dependence between the local intervals is ensured [13]. Note that also non-orthogonal $\psi_{j}(\boldsymbol{r})$ can be used for the interval field series expansion, as e.g., proposed in [5].

The solution of a model $\mathcal{M}$ containing interval or interval field valued uncertain parameter then comes down to finding a solution set $\tilde{\boldsymbol{y}}$ containing the extreme realizations of $\boldsymbol{y}^{m}$ given the hyper-cubic parameter uncertainty. In general, $\boldsymbol{y}^{m}$ is located on a non-convex manifold in $\mathbb{R}^{d}$, since $\mathcal{M}$ provides coupling between $y_{i}^{m}, i=1, \ldots, d$. As such, a general solution to the interval problem is $N P$-hard. As a remediation, the solution set $\tilde{\boldsymbol{y}}$ is commonly approximated by an uncertain realization set $\tilde{\boldsymbol{y}}^{m}$, which 
is obtained by propagating $q$ well selected deterministic realizations $\boldsymbol{x}_{j}$ of the hyper-cubic uncertain input parameters $\boldsymbol{x}^{I}$ :

$$
\tilde{\boldsymbol{y}}^{m}=\left\{y_{j}^{m} \mid y_{j}^{m}=\mathcal{M}\left(\boldsymbol{x}_{j}^{m}\right) ; \boldsymbol{x}_{j}^{m} \in \boldsymbol{x}^{I} ; j=1, \ldots, q\right\}
$$

In general, $\tilde{\boldsymbol{y}}^{m} \approx \tilde{\boldsymbol{y}}$ when $q$ is taken sufficiently large, or sampled intelligently. When $\mathcal{M}$ is a strictly monotonic FE model, the Transformation Method [14] provides an exact mapping from $\boldsymbol{x}^{I}$ to $\tilde{\boldsymbol{y}}$, albeit needing $2^{k}$ deterministic model evaluations for the solution of a single interval problem. Alternatively, recent work has been focused on finding the solution to this problem using extensions of the concept of affine arithmetic [15], or global optimization approaches in order to construct an hyper-cubic approximation $\boldsymbol{y}^{m, I}$ of $\tilde{\boldsymbol{y}}$ [2].

A mathematical handle to the boundaries of $\tilde{\boldsymbol{y}}^{m}$ is provided by the convex hull $\mathcal{C}^{m}(\tilde{\boldsymbol{y}})$ of the realization set $\tilde{\boldsymbol{y}}^{m}$. The convex hull $\mathcal{C}^{m}(\tilde{\boldsymbol{y}})$ can be considered as a set of vertices bounding the uncertain realization set:

$$
\mathcal{C}^{m}(\tilde{\boldsymbol{y}})=\left\{\sum_{j=1}^{q} \beta_{j} y_{j}^{m} \mid\left(\forall j: \beta_{j} \geq 0\right) \wedge \sum_{j=1}^{q} \beta_{j}=1 ; y_{j}^{m} \in \tilde{\boldsymbol{y}}^{m}\right\}
$$

with $\beta$ a vector of weighting factors, such that all elements $\beta_{j}$ are non-negative and sum to one. Alternatively, it can also be represented as a set containing $h_{s} d$-dimensional half-spaces representing the linear inequalities that describe boundaries of $\mathcal{C}^{m}(\tilde{\boldsymbol{y}})$ :

$$
\mathcal{C}^{m}(\tilde{\boldsymbol{y}}) \equiv \boldsymbol{A}_{s}\left(\boldsymbol{y}^{m}\right)^{T}-\boldsymbol{b}_{s} \geq 0
$$

with $\boldsymbol{A}_{s} \in \mathbb{R}^{h_{s} \times d}, \boldsymbol{b}_{s} \in \mathbb{R}^{h_{s}}$ and $h_{s}$ the number of half-spaces that are needed to describe $\mathcal{C}^{m}(\tilde{\boldsymbol{y}})$.

\section{Interval field Quantification.}

Identification of $\boldsymbol{\alpha}^{I}$ is performed by minimising a cost function $\delta\left(\boldsymbol{\alpha}^{I}\right)$, which describes the discrepancy between $\tilde{\boldsymbol{y}}^{m}$ and a set of experimentally obtained responses $\tilde{\boldsymbol{y}}^{e}$ :

$$
\delta\left(\boldsymbol{\alpha}^{I}\right)=\left(\Delta\left(\mathcal{V}^{e}\right)^{2}+w_{0} \cdot \Delta\left(\mathcal{V}^{o}\right)^{2}+\Delta c^{2}\right)
$$

with:

$$
\begin{aligned}
& \Delta \mathcal{V}^{e}=1-\frac{\mathcal{V}^{m}\left(\boldsymbol{\alpha}^{I}\right)}{\mathcal{V}^{e}} \\
& \Delta \mathcal{V}^{o}=1-\frac{\mathcal{V}^{o}\left(\boldsymbol{\alpha}^{I}\right)}{\mathcal{V}^{e}} \\
& \Delta c=\left\|c_{e}-c_{m}\left(\boldsymbol{\alpha}^{I}\right)\right\|_{2}
\end{aligned}
$$

with $\mathcal{V}^{e}$ and $\mathcal{V}^{m}$ the Lebesgue measures of the convex hulls $\mathcal{C}^{e}(\tilde{\boldsymbol{y}})$ and $\mathcal{C}^{m}(\tilde{\boldsymbol{y}})$, computed over $\tilde{\boldsymbol{y}}^{e}$ and $\tilde{\boldsymbol{y}}^{m}$ respectively. Parameters $c_{e}$ and $c_{m}$ are the geometrical centres of mass of these respective convex hulls. $\mathcal{V}^{o}$ is the multidimensional volume of the overlap between $\tilde{\boldsymbol{y}}^{e}$ and $\tilde{\boldsymbol{y}}^{m}$. The parameter $w_{0}$ allows the analyst to enforce that $\mathcal{C}^{e}(\tilde{\boldsymbol{y}})$ is fully encompassed by $\mathcal{C}^{m}(\tilde{\boldsymbol{y}})$. The interval vector $\boldsymbol{\psi}_{i}(\boldsymbol{r})$, used for the construction of $\boldsymbol{x}^{I}(\boldsymbol{r})$, is finally determined as :

$$
\boldsymbol{\alpha}^{I, *}=\operatorname{argmin}\left(\delta\left(\boldsymbol{\alpha}^{I}\right)\right)
$$

For a thorough discussion of this method, the reader is referred to [12] and [13]. A comparison of this method with Bayesian model updating on a high-dimensional case study is given in [9]. 


\section{Case Study: Laser Sintered PA-12 Components}

The presented methods are applied to the study of local uncertainty in Young's modulus of a PA-12 component that is produced using Laser Sintering. For the construction of the measurement data set, the specimen that is shown in Fig. 1 is constructed using standard commercial production parameters. Specifically, a total of 12 parts are constructed in three orientations: Edge, Flat and Upright, where Upright denotes that the specimen is constructed with its longitudinal axis perpendicular to the layer plane $(x, y)$. Edge and Flat specimens are respectively constructed with their front surface in the $(x, z)$ and $(x, y)$ plane, where $z$ denotes the vector perpendicular to the layers of the part, and $x$ and $y$ correspond to the Cartesian coordinate system of the LS machine. These specimens are loaded in displacement controlled mode in a tensile testing machine until a load of 1500N. Digital Image Correlation is applied during the tensile testing to measure the strain fields that correspond to each load step. The parameters that are used for the DIC calculations are listed in table 1.

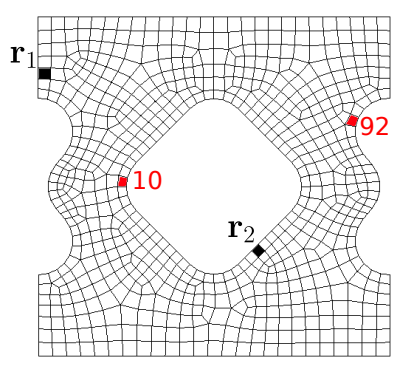

Fig. 1: Considered test geometry

A finite element model representing the elastic constitutive behaviour of these parts under uniaxial loading is constructed in NX Nastran using 775 quadratic quadrilateral elements. The corresponding domain discretization is illustrated in Fig. 1. Based on previous work of the authors, an isotropic constitutive model is assumed. Herein the nominal value for Young's modulus and Poisson's ratio are taken to be $1632 \mathrm{MPa}$ and 0.41 .

Table 1: Adopted parameters for the DIC measurements

\begin{tabular}{llll}
\hline Parameter & Value & Parameter & Value \\
\hline Dynamic range & 8 bit & Step size & $7 \mathrm{px}$ \\
Matching Criterion & ZNSSD & Subset size & $25 \mathrm{px}$ \\
Interpolation & Bicubic splines & Strain window & $15 \mathrm{px}$ \\
Shape function & Affine & Virtual Strain Gage & $91 \mathrm{px}$ \\
Prefiltering & Gaussian & & \\
\hline
\end{tabular}

Two basis functions for the interval field series expansion are constructed, based on a prior expert estimate of the spatial nature of the uncertainty. They are specifically constructed using inverse distance weighting interpolation [13]. The corresponding control points $\boldsymbol{r}_{1}$ and $\boldsymbol{r}_{2}$ are shown in Fig. 1, and they correspond to zones with a difference in cross-sectional area, as previous work of the authors [19] shows that this influences the local stiffness of Laser Sintered parts. As such, the interval field quantification problem reduces to finding the correct interval scalars using the optimisation problem introduced in eq. (8).

Prior to applying the identification and quantification procedure, some operations on the DIC measurement data are necessary. First, since not all tested specimens can be placed exactly the same with respect to the DIC camera's, the coordinate systems of all measured strain fields should be aligned. This operation also needs to be performed between the aligned measured strain fields and the predicted strain field of the FE model. This alignment is obtained by algorithmically detecting the edges in the 
images via a combination of Otsu's method and evaluations of the Laplacian over the image greyvalues, and subsequently fitting those detected edges of the parts in a least-squares sense. Secondly, since the number of measured responses in the DIC data varies from measurement to measurement, and are furthermore higher-dimensional as compared to the strain fields that are predicted by the FE model, interpolation has to be performed. This is specifically obtained by performing a bi-cubic spline interpolation to each measured strain field, and fitting the measured strain fields to the centres of gravity of each local element of the FE model, as shown in Fig. 2. In this interpolation, care is taken that no extrapolation of measured strain values towards nodes of the FE model is made since this has a detrimental effect on the accuracy.

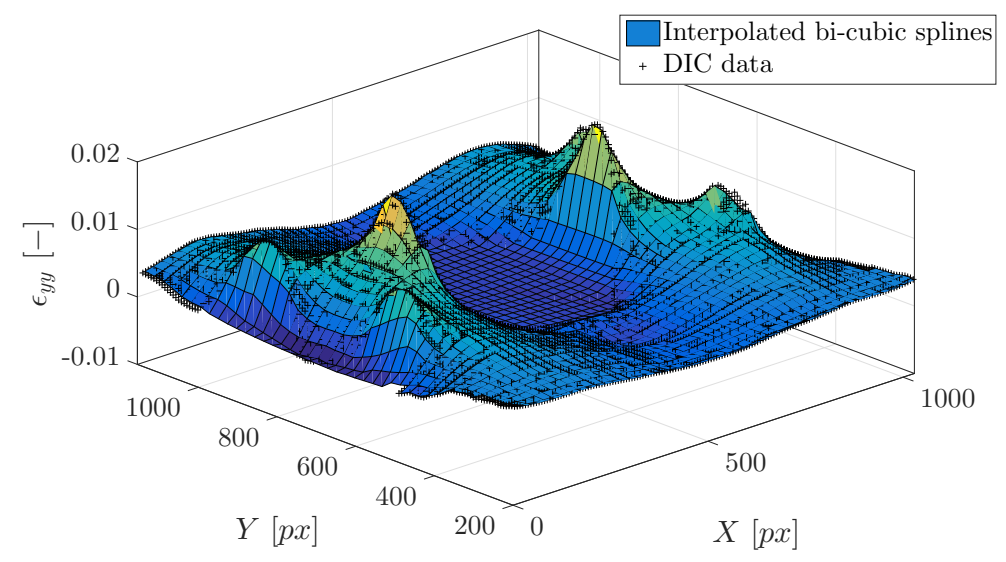

Fig. 2: Bi-cubic spline interpolation of the measured longitudinal strain field

Finally, since the computational cost of obtaining a convex hull scales exponentially with the dimension over which it has to be computed, a reduction of the dimension of the DIC and finite element predicted data is pre-emptive before applying the quantification procedure. This reduction is based on the subset selection method presented in [12], combining information on the sensitivity of the convex hull Lebesgue measure towards the interval field and its aspect ratio. Based on this method, two longitudinal strain components are selected to steer the quantification procedure. Specifically, the longitudinal strains $\epsilon_{y y, 10}$ and $\epsilon_{y y, 92}$ (i.e., the strains on elements 10 and 92) are selected since they provide the highest amount of information for the quantification. These elements are also indicated in Fig. 1.

The solution of eq. (8) is obtained by applying an iterative Newton-type optimisation algorithm with BFGS approximation of the Hessian. Starting from an initial guess (shown in blue), a solution was obtained after 29 iterations of the solver. The quantified interval scalars corresponding to the two base functions are respectively $[1.58 ; 2.15] \cdot 10^{6} \mathrm{MPa}$ and $[1.81 ; 2.35] \cdot 10^{6} \mathrm{MPa}$, which agrees rather well with the results presented in [19]. It can be noted that the upper bounds of these intervals are rather high with respect to literature, which is possibly due to excessive noise in the DIC measurements. Further work however is needed to assess this issue critically. Fig. 3 shows the strains at the selected elements for both the measurements, some initial estimate on the data, and the identified interval field. From this figure, it is clear that the quantified interval field is highly capable of predicting the uncertainty in the strain at those selected responses.

\section{Conclusions}

This paper applies a new method for the quantification of spatial uncertainty to a realistic case using real experimental data. Specifically, the spatial uncertainty in a PA-12 component, produced via Laser Sintering is studied. Hereto, a test population consisting of 12 parts is produced and each part is tested using Digital Image Correlation. A method to automatically process these high dimensional data in terms of automated coordinate system alignment and interpolation to the elements of the FE model is 


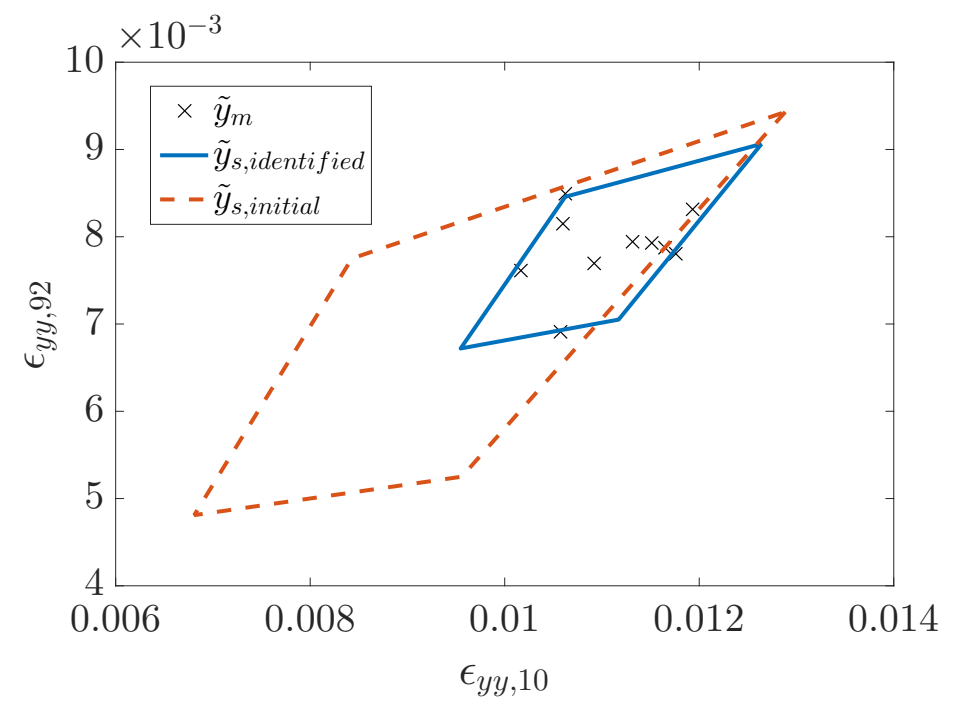

Fig. 3: Two-dimensional cross-section of $\mathcal{C}^{e}(\tilde{\boldsymbol{y}})$ and $\mathcal{C}^{m}(\tilde{\boldsymbol{y}})$

presented. It is shown that an accurate estimation is obtainable that is also objective with respect to the interval scalars in the interval field series expansion. However, in this study, still subjectivity is present in the definition of the basis functions and the interval series expansion order. Furthermore, some overconservatism with respect to literature values for Laser Sintered PA-12 components is present. Further work will therefore focus on objectively quantifying the basis functions and interval field series order expansion using full-field strain measurement data. Possibly, this might also resolve the issue with over-conservatism in the model's responses.

\section{Acknowledgements}

The authors would like to acknowledge support of the Flemish Research Foundation (FWO) in the framework of the project "HiDIF: High Dimensional Interval Fields" (project number G0C2218N).

\section{References}

[1] G. Stefanou. The stochastic finite element method: Past, present and future. Computer Methods in Applied Mechanics and Engineering, 198(9-12) (2009), 1031-1051.

[2] D. Moens, \& M. Hanss. Non-probabilistic finite element analysis for parametric uncertainty treatment in applied mechanics: Recent advances. Finite Elements in Analysis and Design, 47(1) (2011), 4-16

[3] M. Beer, S. Ferson, \& V. Kreinovich. Imprecise probabilities in engineering analyses. Mechanical Systems and Signal Processing, 37(1-2) (2013), 4-29.

[4] W. Betz, I. Papaioannou, \& D. Straub. Numerical methods for the discretization of random fields by means of the Karhunen-Loève expansion. Computer Methods in Applied Mechanics and Engineering, 271 (2014), 109-129.

[5] W. Verhaeghe, W. Desmet, D. Vandepitte \& D. Moens. Interval fields to represent uncertainty on the output side of a static FE analysis. Computer Methods in Applied Mechanics and Engineering, 260 (2013), 50-62.

[6] F. Fedele, R. L. Muhanna, N. Xiao, R. L. Mullen, Interval-Based Approach for Uncertainty Propagation in Inverse Problems, Journal of Engineering Mechanics 4 (1) (2014) 1-7. 
[7] S.-E. Fang, Q.-H. Zhang, W.-X. Ren, An interval model updating strategy using interval response surface models, Mechanical Systems and Signal Processing 60-61 (2015) 909-927.

[8] H. H. Khodaparast, J. E. Mottershead, K. J. Badcock, Interval model updating with irreducible uncertainty using the Kriging predictor, Mechanical Systems and Signal Processing 25 (4) (2011) 1204-1206.

[9] M. Broggi, M. Faes, E. Patelli, Y. Govers, D. Moens, M. Beer. Comparison of Bayesian and Interval Uncertainty Quantification: Application to the AIRMOD Test Structure. 2017 SSCI Proceedings (2017), 1684-1691.

[10] E. Patelli, Y. Govers, M. Broggi, H. M. Gomes, M. Link \& J.E. Mottershead. Sensitivity or Bayesian model updating: a comparison of techniques using the DLR AIRMOD test data. Archive of Applied Mechanics, 87(5) (2017), 905-925.

[11] M. Faes, J. Cerneels, D. Vandepitte \& D. Moens. Identification of Interval fields for spatial uncertainty representation in Finite Element models. Proceedings of the European Congress on Computational Methods in Applied Sciences and Engineering: Vol. 7. European Congress on Computational Methods in Applied Sciences and Engineering. Crete Island, Greece, 5-10 June 2016

[12] M. Faes, J. Cerneels, D. Vandepitte \& D. Moens. Identification and quantification of multivariate interval uncertainty in finite element models. Computer Methods in Applied Mechanics and Engineering, 315 (2017), 896 - 920.

[13] M. Faes \& D. Moens. Identification and quantification of spatial interval uncertainty in numerical models. Computers and Structures, 192 (2017), 16-33.

[14] M. Hanss. The transformation method for the simulation and analysis of systems with uncertain parameters. Fuzzy Sets and Systems, 130(3) (2002), 277-289.

[15] A. Sofi \& G. Muscolino. Static analysis of Euler-Bernoulli beams with interval Young's modulus. Computers and Structures, 156 (2015), 72-82.

[16] A. Sofi, G. Muscolino \& M. Zingales. One-dimensional heterogeneous solids with uncertain elastic modulus in presence of long-range interactions: Interval versus stochastic analysis. Computers and Structures, 122 (2013), 217-229.

[17] D. Wu, and W. Gao Uncertain static plane stress analysis with interval fields. International Journal for Numerical Methods in Engineering, 110(2017) 1272-1300.

[18] C. B. Barber, D. P. Dobkin \& H. Huhdanpaa. The quickhull algorithm for convex hulls. ACM Transactions on Mathematical Software, 22(4) (1996), 469-483.

[19] M. Pavan, M. Faes, D. Strobbe, B. Van Hooreweder, T. Craeghs, D. Moens, \& W. Dewulf. On the influence of inter-layer time and energy density on selected critical-to-quality properties of PA12 parts produced via laser sintering. Polymer Testing, 61 (2017), 386-395. 